\title{
Assessment of the Common Cause Failure(CCFs) effect on Safety Instrumented System(SIS) by usign the Fault Tree Analysis (FTA) method
}

\author{
Metatla Hassina ${ }^{(1)^{*}}$, Rouainia Mounira ${ }^{(2)}$ \\ ${ }^{(1)}$ Department of technology .University 20août 1955-Skikda, Algeria. \\ (2) Department of petrochemistry and process engineering. University 20août 1955-Skikda, Algeria. \\ "Corresponding author e-mail address:metatla.hassina@yahoo.fr,h.metatla@univ-skikda.dz
}

\begin{abstract}
The reliability of the safety-instrumented system (SIS) has received a lot of attention during the past decade, with the emergence of the new standards such as International Electrotechnical Commission IEC61508, and IEC61511, that provides a general framework for the design and implementation of these safety barriers. Among the problems influencing on the global SIS reliability: Common Cause Failure (CCF), which contributes too many accidents, that has a negative impacts, so it must be considered in the risk and reliability assessment for these systems. The aim of this work is to focus on the effects of common cause failures (CCFs) on the reliability of a SIS, by implementing a comparative SIS dependability study with and without consideration the CCFs, using the beta factor model, and the fault tree analysis (FTA) method.
\end{abstract}

Keywords: Common cause failure (CCF), Beta-factor- model, Fault Tree Analysis, Minimal cuts sets, Dependents and independents failures, and safety-instrumented system.

\section{INTRODUCTION}

Safety instruments systems (SIS) are vital safety barriers to reduce the probability of the hazardous events and mitigate their consequences. The SIS has been widely used in many several industries. A SIS is regarded as a low demand system when the frequency of demands is no greater than once per year and no greater than twice the proof test frequency. Otherwise, the SIS is regarded as a high demand system. Low demand systems are very different from the high demand systems not only the frequency of failure on demand, but also the methods used to analyze the reliability of SIS [1].

Redundancy is often introduced in SIS for higher reliability, although redundancy has many benefits, their negative effects cannot be ignored, redundancy induces common cause failure (CCFs) to the SIS; therefore the CCFs are a big threat to the reliability of systems, which contributes too many major accidents, that has major negative impacts. So is very important to consider CCFin the risk and reliability assessment for the whole life cycle especially in the design phase. Until now, it is impossible to avoid the CCFs, hence what we can do to reduce common causes failure as much as possible. An important part of SIS management is therefore to assess and implement measures to reduce the influence of CCFs on the global reliability.

CCFs are simultaneous failures of multiple components within a system due to a common-cause or a shared root cause. CCFs can contribute significantly to the overall system unreliability. Therefore, it is important to incorporate CCFs into the system reliability analysis. CCFs have received considerable attention over several decades, but the main attention has been development of models rather than collecting data to support the models, see, e.g. Hokstad and Rausand [2] for an overview. Reliability modeling of CCFs was introduced in the nuclear industry for about 40 years ago, and early results were presented e.g. in NUREG75/014 [3], Edwards, and Watson [4]. In addition, the aviation industry has given a close attention to CCFs, and more recently the standard IEC61508 for oil and gas industry [1] has pointed out the importance of controlling these failures in order to maintain the integrity of the safety instrumented functions (SIFs). 
There are two common causes' failures modeling methods: explicit modeling and implicit modeling [7]. The Beta factor model belongs to implicit modeling and it is the simplest and widely used model. Many common cause failure models have been developed on the Beta factor model such as: Multiple Greek Letter (MGL), and Alpha Factor Model [5] [6].

The Beta factor model originally developed for two components, later extended to larger systems, it is based on notion that components failures can be divided into two groups: independent failures, and dependent failure of all components. For the Multiple Greek Letter (MGL) model is the Beta factor extension to treat multiple levels of CCFs it is more appropriate for systems with high level of redundancy. The Alpha factor model is a simple expression for exacted distributions of MGL parameters accounting for uncertainties [7].

The oil and gas industry is mainly focusing on CCFs in the design phase of the SIS, while CCFs are given much less attention in the operational phase. In the design phase CCFs causes may be result of inadequate understanding of failure mechanisms and responses, improper selection of hardware components, and so forth. In the operational phase, CCFs causes may be introduced because of improper testing, human errors during operation andmaintenance, and environmental stresses outside the design envelope [8].The aim of this paper is to assess the dependability of the High Integrity Pressure Protection System (HIPPS) with and without consideration of the CCFs by using the Fault Tree Analysis (FTA) method. The HIPPS is used in oil and gas fields for a long time his majors functions are: to measure pressure, to evaluate pressure signals, and stopping flow. In order to do this the pressure must be sensed and converted to electrical signal, and the signal must be transmitted for evaluation [9].

The rest of this article is organized as follow: in section2, SIS description is presented, then in section3 the classification of SIS failures' are explained, in the next section, the fault tree analysis method that is used to assess theHIPPS reliability is provided. In section5, we compute the parameter of the HIPPS dependability with and without CCFs, and the simulation results are discussed. We conclude in section 6 by giving some recommendations and perspectives.

\section{SAFETY INSTRUMENTED SYSTEM} (SIS)

Safety instrumented system (SIS) are increasingly used across a wide range of industries to perform safety instrumented functions (SIFs). These computer are generally composed on three independent parts: detection (sensors), treatment (logic solver), and actuation items.

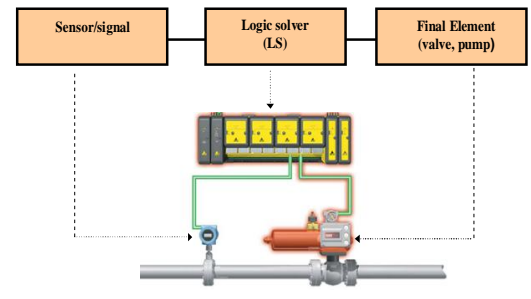

Fig.1 Safety Instrumented System structure.

- Sensor/Input interface such as: transmitters, sensors.

- Logic Solver: such as programmable Logic Controller (PLC), micro processor

- Final element/Output interface such as: valve, pump.

- Sensors: which monitor the evaluation of the physical or chemical parameters representing the process performance (temperature, pressure, flow, level....etc.). If at least one of these parameters exceeds a set level and remains there, this deviation is detected and the alarm is generated by the logic solver program.

- Logic solver: the purpose of this component of SIS is to determine what action is to be taken based on the information gathered. Highly reliable logic solvers are used which provide both fail-safe and tolerant operation. It is typically a controller that reads signals from the sensors and executes preprogrammed actions to prevent a hazard by providing an output to the final control elements.

- Final control element: it implements the action determined by the logic system. This final control element is typically a pneumatic actuated on-off valve operated by 
solenoid valves. It is imperative that all three elements of the SIS system function are designed in order to safety isolate the process plant in the event of an emergency [1].

\section{SIS FAILURES'}

The standard IEC 61508(2010) classified failures into random hardware failure, and systematic failure based on the causes of failure. Random hardware failure can also be called physical failure. While systematic failure is not physical failure.

\section{- Systematic failure:}

A failure related in a deterministic way to a certain cause, which can only be eliminated by a modification of the design or the manufacturingprocess, operational procedures, documentation, or other relevant factors.

- Human factor: or human errors include operational and installations failures and all failure performed during operational, testing/maintenance and repair as well as during commissioning installations and modifications. Such failures can materialize one being introduced, but may often remain inherent in the system for a long period and materialize during an actual demand [1][2].

- $\quad$ Radom hardware failure: this kind of failure occurring at a random time, this result from one or more of the possible degradation mechanisms in the hardware. This failure can further be divided into aging failure and stress failure. Aging failures are failures occurring because of design scope, whereas Stress failures are failures that occur due to excessive stress of the item, which may be caused by human error when operating. The detailed failure classification is shown in figure2.

\begin{tabular}{|l|l|l|}
\hline Systematic & Hardware & Human factor \\
\hline $\begin{array}{l}\text { Software faults. } \\
\text { Installations } \\
\text { failure. }\end{array}$ & $\begin{array}{l}\text { Aging failure. } \\
\text { Environment. } \\
\text { (Vibration } \\
\text { Temperature, } \\
\text { Corrosion). }\end{array}$ & $\begin{array}{l}\text { Wrong operation. } \\
\text { Maintenance } \\
\text { induced. }\end{array}$ \\
\hline
\end{tabular}

Fig. 2 CCFs causes'.
Through the classification of failure, it is easy to understand the failures and asses the SIS reliability. The following methods are used to analyze the reliability of these barriers: Markov method, Fault Tree Analysis (FTA), Reliability Block Diagram (RBD), Risk graph, and Layer of protection analysis (LOPA).

A Markov method is dynamic method suitable for a high- demand systems, while fault tree analysis and reliability block diagrams are usually used for low-demand systems [19].

\subsection{Dependent or common cause failure (CCF)}

Because of the high level of redundancy, the system may be influenced by common cause failure (CCF). The CCF is defined as dependent failure in which two or more component fault state exists simultaneously, or within a short time interval, and it is a direct result of a shared cause. Such failure may be caused by design or material deficiency error during installation or maintenance, or by environmental condition.

Part"4" of the standard IEC61508 defines a common cause failure as a failure which is the result of one or more events, causing concurrent failures of two or more separate cannels in a multiple channel system, leading to system failure. These failures have a significant effect on reliability and safety of a SIS, and therefore must be considered in the reliability and safety model [1] [2].

\subsection{Common cause failure modeling}

There are two kinds of modeling for CCF:

Explicit modeling: when the specific causes of CCFs can be identified and the causes are dependent failure, it is better to model CCFs explicitly. The basic events in a fault tree model are considered as specific causes. The explicit causes are human error, utility failure or environmental events. One of the advantages of the explicit modeling is that all the root causes of CCFs can be identified.

Implicit modeling: when the causes of CCFs are difficult to be identified or cannot be identified, then the CCFs will be modeled implicitly. The limitation of the implicit modeling is that the causes of the failure cannot be identified clearly [7]. 


\subsection{Beta factor model}

The $\beta$-factor model was proposed by Fleming in 1975. Nowadays, it is still a widely used CCF model because of its simplicity. It can be explained by a simple example: if a system includes $\mathrm{n}$ identical components and all have a constant failure rate $\lambda$, two kinds of failure rates are introduced:

$\lambda_{\mathrm{i}}$ : is the independent failure rate which will not cause other components failure.

$\lambda_{c}$ : is the common cause failure rate which denotes all the component's failure caused by a shared cause.

Therefore the total failure rate for one component is:

$$
\lambda=\lambda_{i}+\lambda \mathbf{c}
$$

For two components, the relationship of the $\mathrm{CCF}_{\mathrm{s}}$ and independent failures is show in figure3.

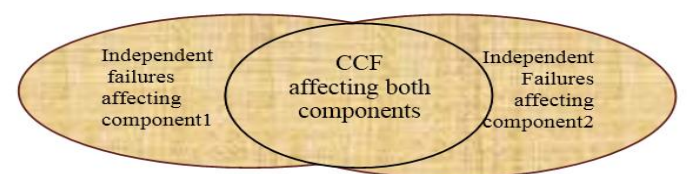

Fig. 3 Relationship between independent failure and CCF.

Beta-factor denotes the fraction of CCFs among all failures of one component. Betafactor can be expressed as:

$$
\beta=\frac{\lambda c}{\lambda}, \text { so }: \lambda c=\beta \lambda
$$

Therefore: $\boldsymbol{\lambda} \mathbf{i}=(\mathbf{1}-\boldsymbol{\beta}) \boldsymbol{\lambda}(3)$

If a component fails, $\beta$ is the probability of $\mathrm{CCF}$, therefore the probability of independent failure is (1- $\beta$ ). The relationship between CCF and independent failure is expressed by $\beta$.

Advantages of beta-factor model:

- It is simple, only one parameter $\beta$ need to be estimated when the data are available.

- It is easy and widely used; some standards (IEC61508) recommend this $\beta$ factor model to assess reliability of SIS.
- Many models are developed based on $\beta$-factor model such as: $\alpha$-factor model, multiple beta- factor models, Multiple Greek Letter (MGL) model [7][8].

\section{FAULT TREE ANALYSIS (FTA) METHOD}

Fault tree analysis (FTA) is a top-down qualitative approach originally used to identify failure in complex systems. A fault tree analysis begins with the 'top event' this is the result of number of basic events that contributes to the system failure or initiates it. The logic of a fault tree is displayed by symbols that represent the basic events and gates that logically relate those events. Each common fault tree symbol represents a type of event or a logical relationship. The fault tree method is used to find combination of failures that may cause problems and helps the analyst focus on one failure type at a time, by identifying which parts of a system are related to a particular failure [5].

Fault tree analysis can be a very powerful tool for analyzing the frequency or probability of an accident. The FTA method cannot only represent the way that events are logically related but can also quantify the probability of those events. Additional analyses allow the determining of the main importance factors [9] in a system:

- Marginal Importance factor (MIF)

- Critical Importance factor (CIF)

- Diagnostic Importance factor (DIF)

- Reliability AchievementWorth (RAW)

- Reliability ReductionWorth (RRW)

The use of these factors is to find the most vulnerable components in the system and to increase the reliability, safety, and maintainability of these critical components, [10][11] and orienting maintenance efforts.

\section{APPLICATION}

The example of our application is a High Integrity Pressure Protection System (HIPPS), which is an SIS system used in the oil and gas industry for a long time. A basic HIPPS is illustrated in figure4. Redundant 2003 voted pressure transmitters detects high pressure and gives a signal to the logic solver. The 2003 voting offers both a level of redundancy and fault tolerance. The logic solver sends signals to the solenoid valves to 
close and acts as barriers again the high pressure [12].

SV1, SV2 are the Solenoid Valves, SDV1, and SDV2: are the Safety Valves, or the barriers valves that are the final elements of the HIPPS. They close and act as barriers to protect against over-pressure. The HIPPS valves have a fail -safe design, meaning that any loss of electric or hydraulic power will cause the valves to close. SDV1 and SDV2 are installed in series with a 1002 voting to meet the requirements of IEC61508. Valve closing is decided to prevent pressure built up in the low rated zone [12][13].

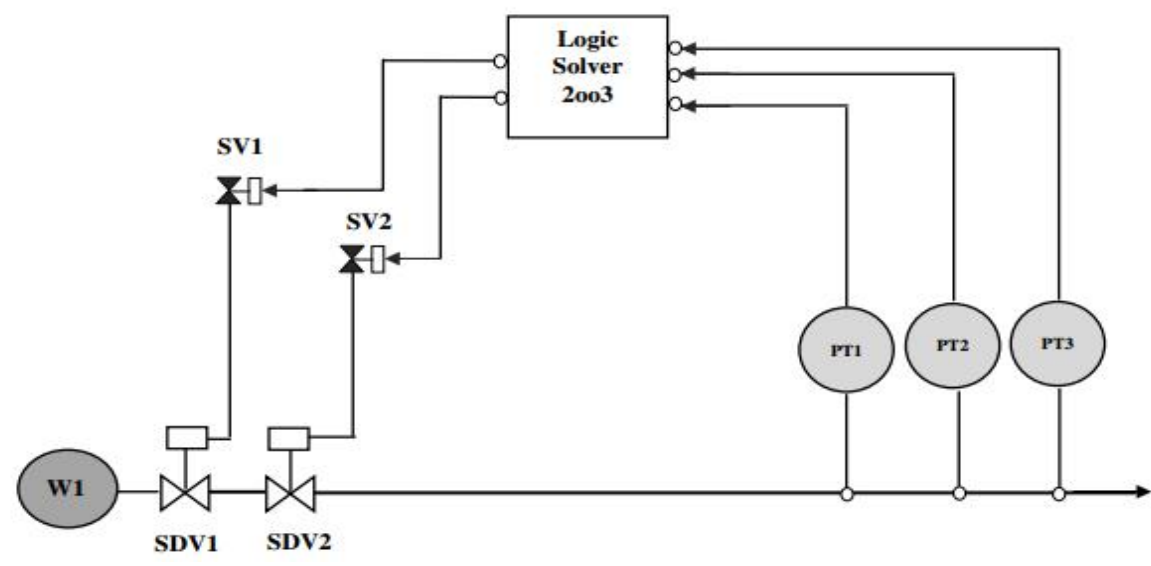

Fig. 4 the HIPPS' Components.

The main objective of this paper is to quantify the HIPPS reliability, and Probability of Failure on Demand: PFD $_{\text {avg }}$ through the use of the Beta factor model and the FTA method, with and without considerationthe common cause failure (CCFs), and to study how the CCFsinfluence onthe value of the HIPPS PFD avg. .

\subsection{Fault Tree Analysis (FTA) of the HIPPS without CCF.}

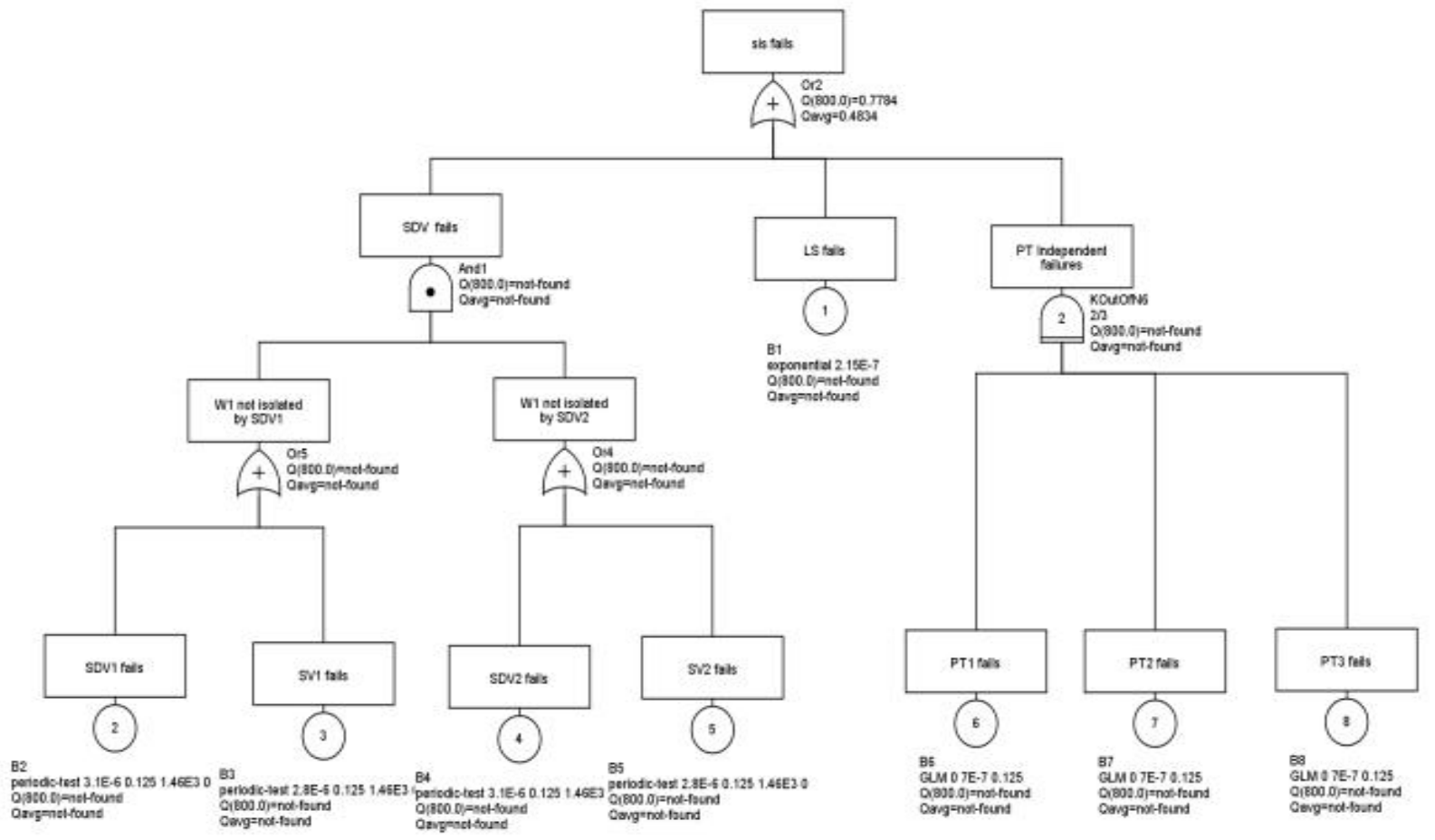

Fig. 5 HIPPS fault tree analysis without consideration the CCFs. 


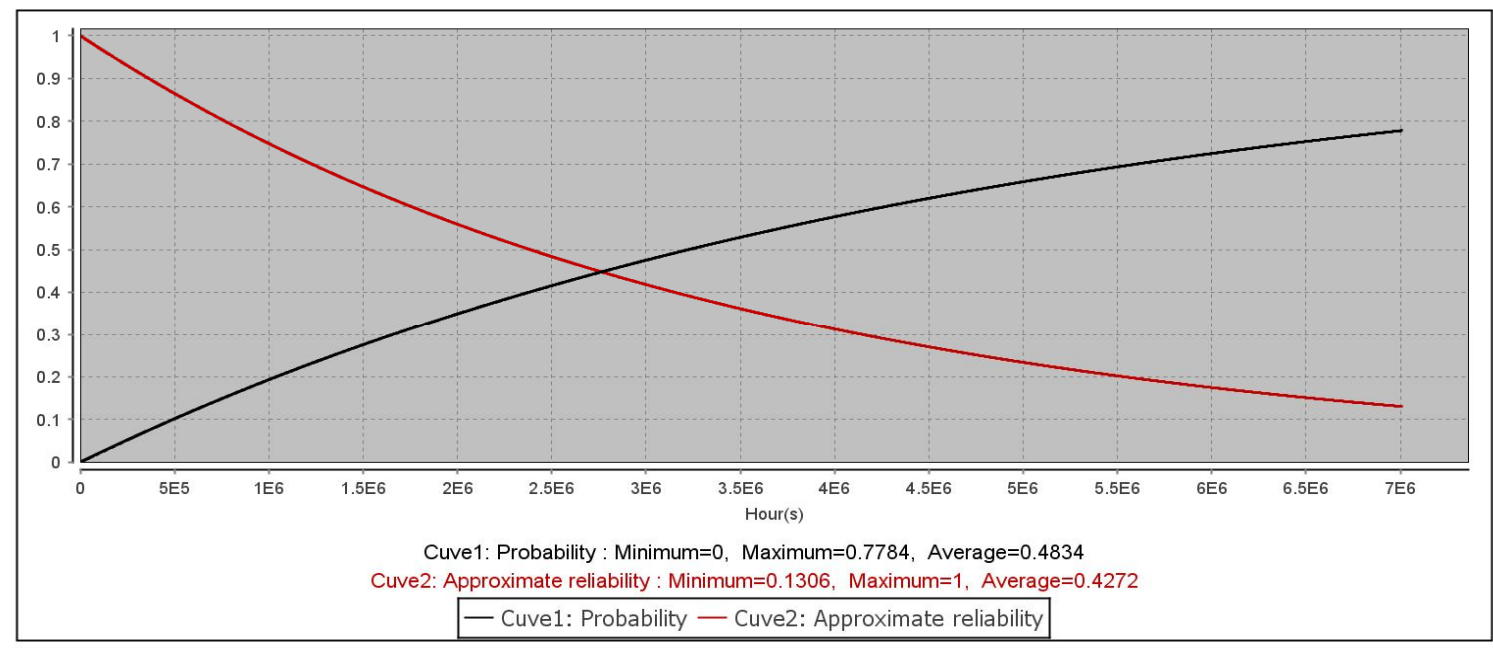

Fig. 6 HIPPS reliability and PFD avg without CCFs.

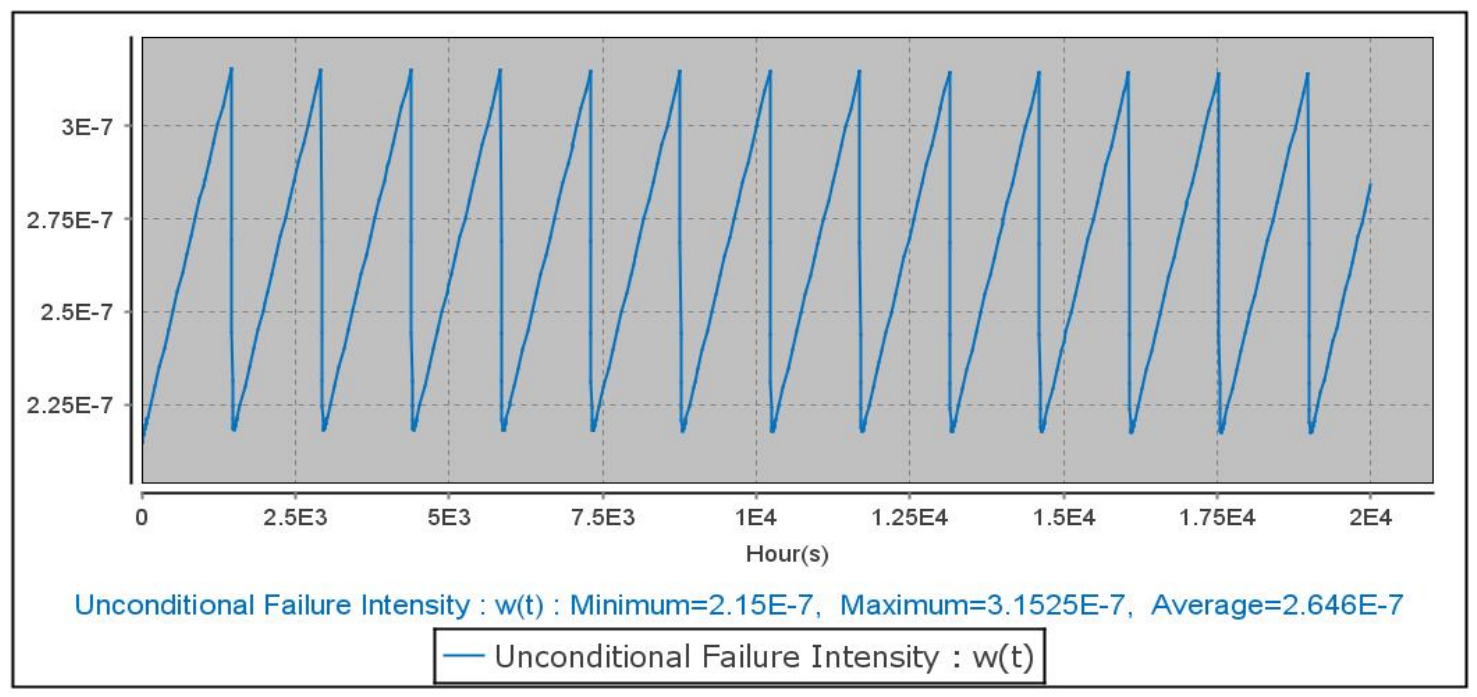

Fig. 7 Unconditional failure intensity of the top event.

-Unconditional intensity of fault $w(t)$, or the frequency of feared event is $w(t)=2.646 E-7$.

Table.1 Minimal cuts set of the HIPPS

\begin{tabular}{|c|c|c|}
\hline \multicolumn{3}{|c|}{ PRODUCTS } \\
\hline \multicolumn{3}{|c|}{ Type= PRODUCTS, Name =sis fails } \\
\hline Order & Products & Probability \\
\hline 1 & LS & $1.8816 \mathrm{E}-3$ \\
\hline 2 & SDV2, SV1 & $1.8422 \mathrm{E}-5$ \\
\hline 2 & SDV1, SDV2 & $2.0391 \mathrm{E}-5$ \\
\hline 2 & SV1, SV2 & $1.6643 \mathrm{E}-5$ \\
\hline 2 & SDV1, SV2 & $1.8422 \mathrm{E}-5$ \\
\hline 2 & PT1, PT2 & $3.136 \mathrm{E}-11$ \\
\hline 2 & PT1, PT3 & $3.136 \mathrm{E}-11$ \\
\hline 2 & PT2, PT3 & $3.136 \mathrm{E}-11$ \\
\hline
\end{tabular}


-They point out the existence of 8 minimal cuts: 7 of order 2 and only 1 of order 1 . The most critically path is Logic Solver (LS), the second are: (SDV2, SV1),(SDV1, SDV2),(SV1, SV2),(SDV1, SV2), and the minimums are:(PT1, PT2),(PT1, PT3),(PT2, PT3).

Table 2 Marginal Importance factor (MIF) for each component.

\begin{tabular}{|l|l|l|}
\hline \multicolumn{3}{|c|}{ Type=MIF, System=sis fails } \\
\hline Time & value & Component \\
\hline $8.76 \mathrm{E} 3$ & $8.522 \mathrm{E}-3$ & SV2 \\
\hline $8.76 \mathrm{E} 3$ & 0.9999 & LS \\
\hline $8.76 \mathrm{E} 3$ & $1.1178 \mathrm{E}-5$ & PT2 \\
\hline $8.76 \mathrm{E} 3$ & $8.5257 \mathrm{E}-3$ & SDV1 \\
\hline $8.76 \mathrm{E} 3$ & $8.5257 \mathrm{E}-3$ & SDV2 \\
\hline $8.76 \mathrm{E} 3$ & $1.1178 \mathrm{E}-5$ & PT1 \\
\hline $8.76 \mathrm{E} 3$ & $8.522 \mathrm{E}-3$ & SV1 \\
\hline $8.76 \mathrm{E} 3$ & $1.1178 \mathrm{E}-5$ & PT3 \\
\hline
\end{tabular}

Table 3 Critical Importance factor (CIF) for each component.

\begin{tabular}{|l|l|l|}
\hline \multicolumn{3}{|c|}{ Type=CIF, System=sis fails } \\
\hline Time & value & Component \\
\hline $8.76 \mathrm{E} 3$ & $1.7783 \mathrm{E}-2$ & SV2 \\
\hline $8.76 \mathrm{E} 3$ & 0.9624 & LS \\
\hline $8.76 \mathrm{E} 3$ & $3.2018 \mathrm{E}-8$ & PT2 \\
\hline $8.76 \mathrm{E} 3$ & $1.9692 \mathrm{E}-2$ & SDV1 \\
\hline $8.76 \mathrm{E} 3$ & $1.9692 \mathrm{E}-2$ & SDV2 \\
\hline $8.76 \mathrm{E} 3$ & $3.2018 \mathrm{E}-8$ & PT1 \\
\hline $8.76 \mathrm{E} 3$ & $1.7783 \mathrm{E}-2$ & SV1 \\
\hline $8.76 \mathrm{E} 3$ & $3.2018 \mathrm{E}-8$ & PT3 \\
\hline
\end{tabular}

Table 4 Diagnostic Importance factor (DIF) for each component.

\begin{tabular}{|l|l|l|}
\hline \multicolumn{3}{|c|}{ Type=DIF, System=sis fails } \\
\hline Time & value & Component \\
\hline $8.76 \mathrm{E} 3$ & $2.179 \mathrm{E}-2$ & SV2 \\
\hline $8.76 \mathrm{E} 3$ & 0.9624 & LS \\
\hline $8.76 \mathrm{E} 3$ & $5.632 \mathrm{E}-6$ & PT2 \\
\hline $8.76 \mathrm{E} 3$ & $2.4119 \mathrm{E}-2$ & SDV1 \\
\hline $8.76 \mathrm{E} 3$ & $2.4119 \mathrm{E}-2$ & SDV2 \\
\hline $8.76 \mathrm{E} 3$ & $5.632 \mathrm{E}-6$ & PT1 \\
\hline $8.76 \mathrm{E} 3$ & $2.179 \mathrm{E}-2$ & SV1 \\
\hline $8.76 \mathrm{E} 3$ & $5.632 \mathrm{E}-6$ & PT3 \\
\hline
\end{tabular}

Table 5 Reliability Achievement Word (RAW) for each component.

\begin{tabular}{|l|l|l|}
\hline \multicolumn{3}{|c|}{ Type=RAW, System=sis fails } \\
\hline Time & value & Component \\
\hline $8.76 \mathrm{E} 3$ & 5.3412 & SV2 \\
\hline $8.76 \mathrm{E} 3$ & $5.115 \mathrm{E}-2$ & LS \\
\hline $8.76 \mathrm{E} 3$ & 1.0057 & PT2 \\
\hline $8.76 \mathrm{E} 3$ & 5.3412 & SDV1 \\
\hline $8.76 \mathrm{E} 3$ & 5.3412 & SDV2 \\
\hline $8.76 \mathrm{E} 3$ & 1.0057 & PT1 \\
\hline $8.76 \mathrm{E} 3$ & 5.3412 & SV1 \\
\hline $8.76 \mathrm{E} 3$ & 1.0057 & PT3 \\
\hline
\end{tabular}

Table 6 Reliability Reduction Word (RRW) for each component.

\begin{tabular}{|l|l|l|}
\hline \multicolumn{3}{|c|}{ Type=RRW, System=sis fails } \\
\hline Time & value & Component \\
\hline $8.76 \mathrm{E} 3$ & 1.0181 & SV2 \\
\hline $8.76 \mathrm{E} 3$ & $2.6577 \mathrm{E} 1$ & LS \\
\hline $8.76 \mathrm{E} 3$ & 1 & PT2 \\
\hline
\end{tabular}




\begin{tabular}{|l|l|l|}
\hline $8.76 \mathrm{E} 3$ & 1.0201 & SDV1 \\
\hline $8.76 \mathrm{E} 3$ & 1.0201 & SDV2 \\
\hline $8.76 \mathrm{E} 3$ & 1 & PT1 \\
\hline $8.76 \mathrm{E} 3$ & 1.0181 & SV1 \\
\hline $8.76 \mathrm{E} 3$ & 1 & PT3 \\
\hline
\end{tabular}

Table 7 Ranking of the components according of their importance factors.

\begin{tabular}{|c|c|c|c|c|}
\hline MIF & CIF & DIF & RAW & RRW \\
\hline LS & LS & LS & LS & LS \\
\hline SDV1=SDV2 & SDV1=SDV2 & SDV1=SDV2 & SDV1=SDV2=SV1=SV2 & SDV1=SDV2 \\
\hline SV1=SV2 & SV1=SV2 & SV1=SV2 & PT1=PT2=PT3 & SV1=SV2 \\
\hline PT1=PT2=PT3 & PT1=PT2=PT3 & PT1=PT2=PT3 & PT1=PT2=PT3 & PT1=PT2=PT3 \\
\hline
\end{tabular}

\subsection{Discussions}

According to the simulation results (Table2, 3, 4, 5, 6, 7), we can say that the FTA method enable us to calculate a range of importance measures: MIF, CIF, DIF, RAW, RRW in the diagnostic stage for the first study (without consideration the CCFs), these factors allow to provide elements that might help to make decision for improving the working of the HIPPS.

According to the tabe.7 the MIF measure the behavior of the global HIPPS reliability, the state of the components. It means to increase the reliability of the HIPPS it is necessary to begin by improving reliability of the logic solver (LS) above all, in second place the safety valves (SDV1, SDV2), and in third place the solenoid valves(SV1, SV2).

The CIF is used to identify the HIPPS weaknesses, or the critical HIPPS components, for the objective of the maintenance optimization, or for to select components for preventive maintenance, and those on which corrective maintenance is enough. The components ranking in table.7 shows that the logic solver (LS) is the element to supervise first and foremost as there is one single treatment unit in the considered system, as it can cause the total fault of HIPPS. The second critical element to consider corresponds to the safety valves (SDV1, SDV2), and the solenoid valves (SV1, SV2), which work in sequential way with (SDV1, SDV2). The last elements to consider are the transmitters PT1, PT2, and PT3. Therefore for the most critical components: logic solver (LS), safety valves
(SDV1, SDV2), solenoid valves (SV1, SV2), they are the object of a systematic or conditional preventive maintenance. While the transmitters $\mathrm{PT}_{\mathrm{i}}$ are the object of a corrective maintenance where intervention is made only after fault, since their breakdown would have not enough influence.

For DIF: this factor holds the name and the usefulness in the diagnosis of reasons of faults of the system, this indicator is very precious in the stage of design, according to the simulation results in table 3 and table4, we can notice that the CIF and DIF give the same classifications especially for the most critical elements (LS, SDV1, SDV2, SV1, and SV2).

The classification of the components showed in the table.5, us informs that the RAW has allowed the extraction of the elements of which are translated by a strong increase of risk.

RRW: the classification in the table.6 gives a selection of the components, which are the best candidates for efforts aiming to improve the total HIPPS reliability. RAW and RRW gives the same order of classification.

The: MIF, CIF, DIF, RAW, RRW allow to provide elements that might help to make decision for improving the working of the HIPPS, they constitute indicators of useful static performances under the hypothesis of binary and independent component for an architecture defined in priori. These factors are an efficient tool in reliability studies, and risk analysis, maintenance optimization, system exploitation and design. 


\subsection{Fault Tree Analysis (FTA) of the HIPPS with CCF using the beta-factor model.}

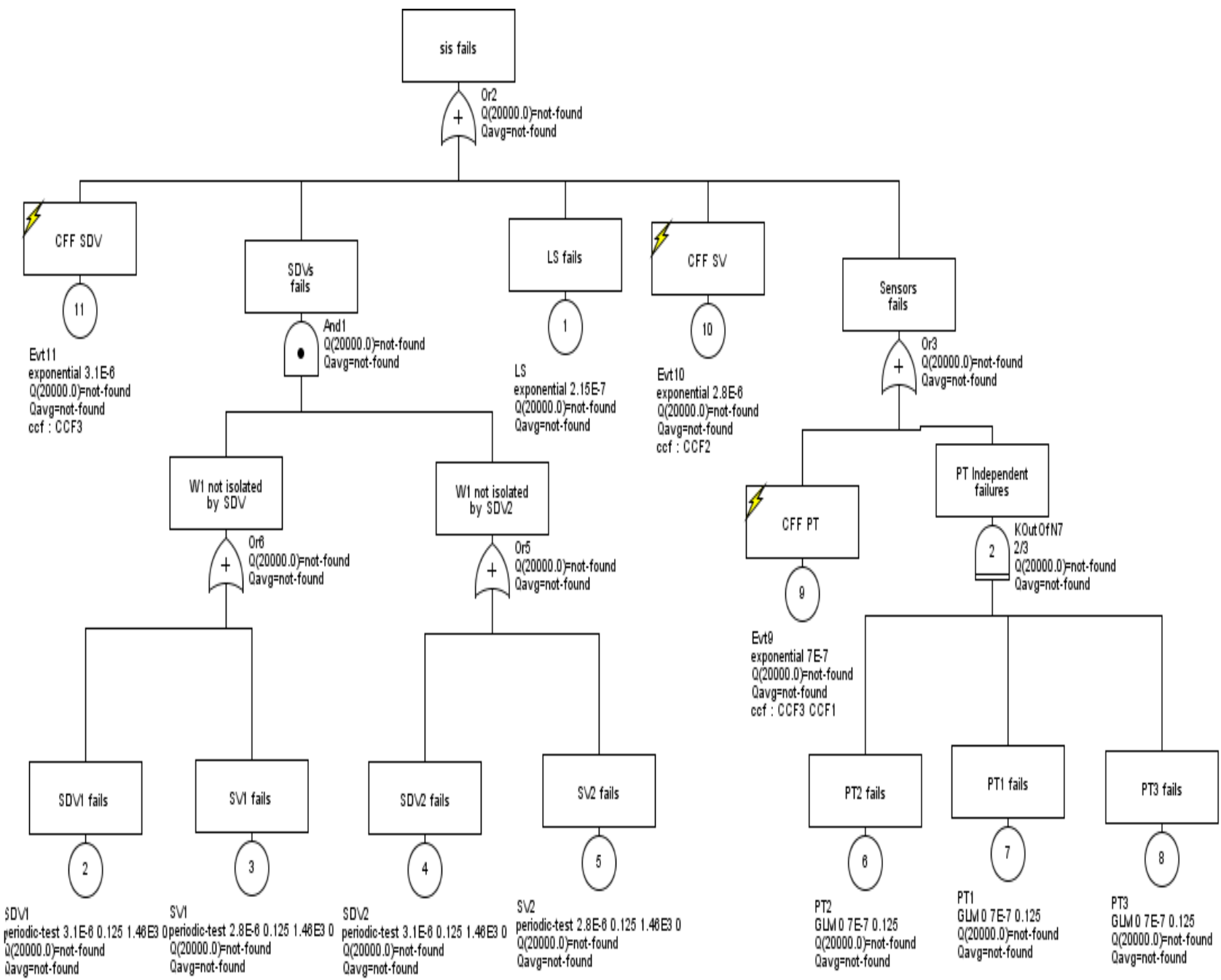

Fig. 8 Fault tree of the HIPPS with consideration the CCFs.

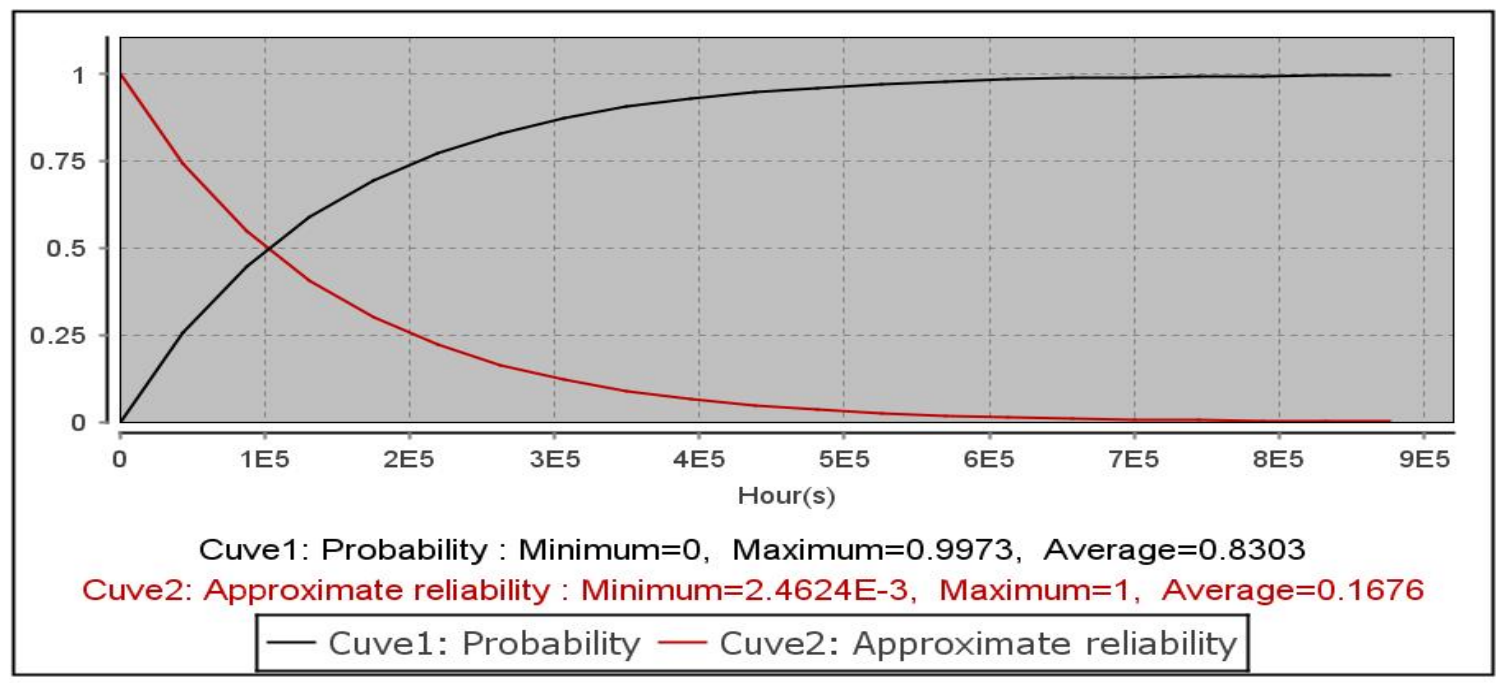

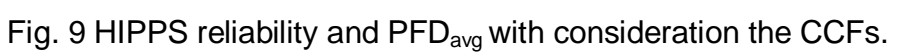




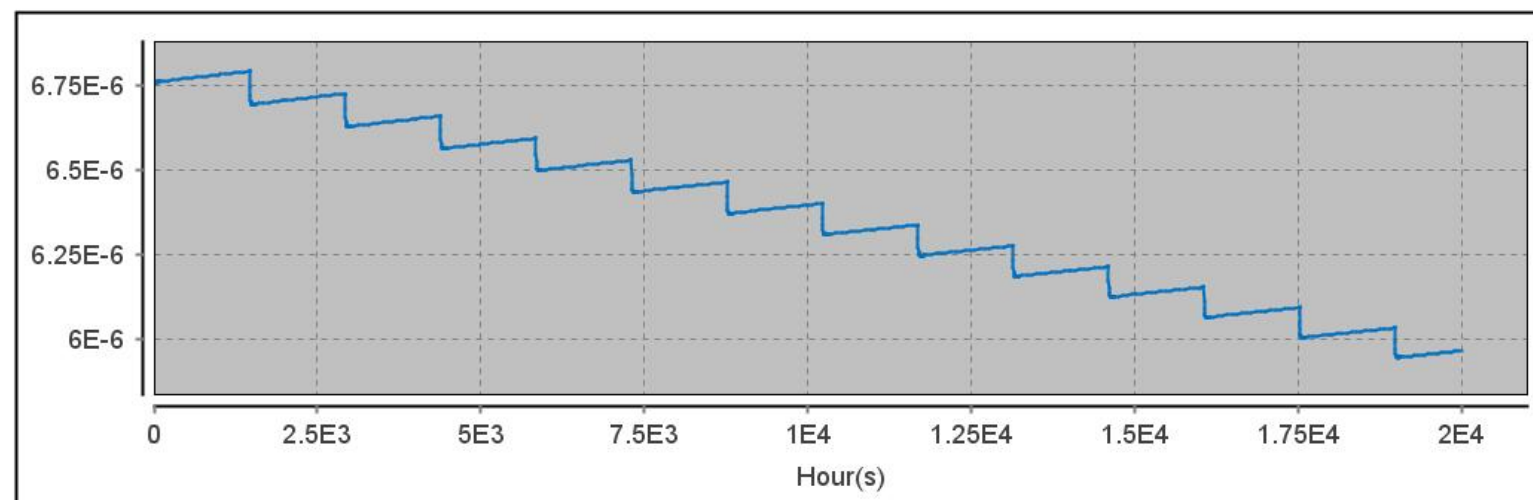

Unconditional Failure Intensity $: w(t):$ Minimum=5.9465E-6, Maximum=6.7915E-6, Average=6.3688E-6

$$
\text { - Unconditional Failure Intensity :w(t) }
$$

Fig. 10 Unconditional failure intensity of the top event.

Unconditional intensity of fault $w(t)$ or the frequency of feared event by consideration of the CCF, this is given as: $W(t)=6.3688 E-6$.

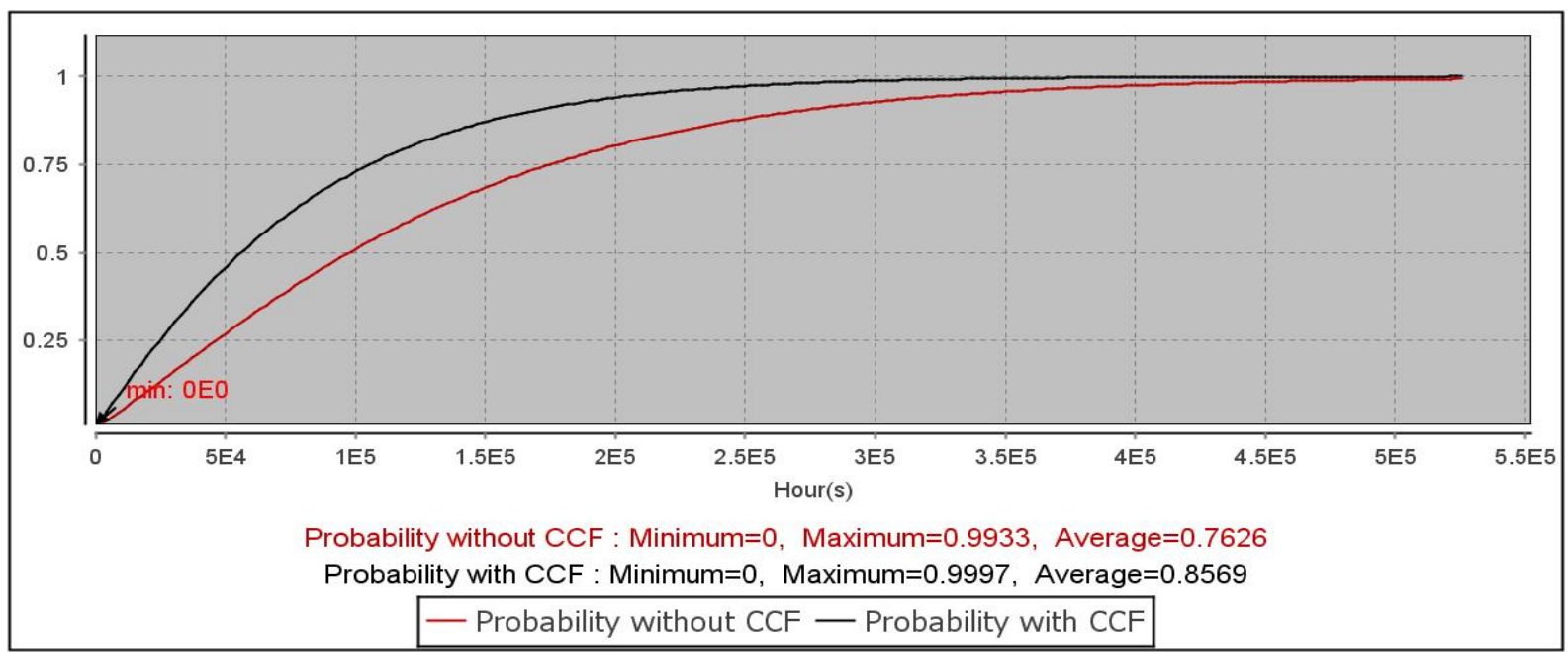

Fig. 11 Curve of the HIPPS PFD avg with and without CCFs.

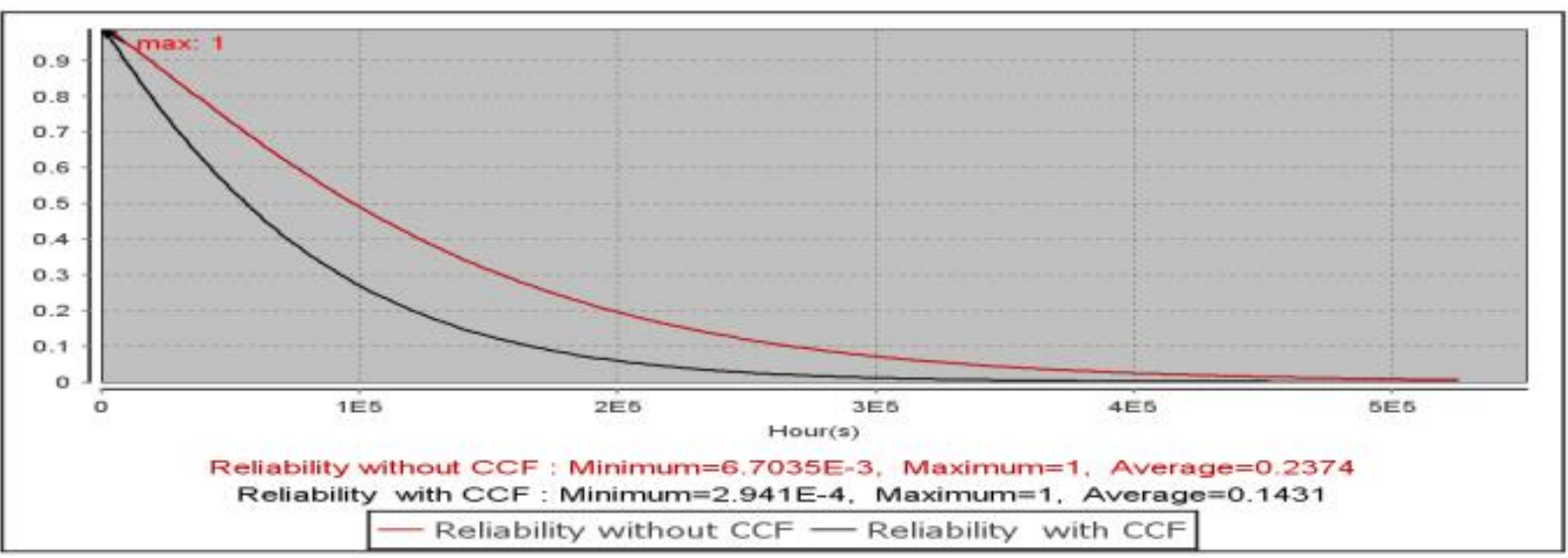

Fig. 12 Curve of the HIPPS reliability with and without CCFs. 
Table 8 Evaluation of HIPPS reliability and PFDavg.

\begin{tabular}{|l|l|l|l|l|l|}
\hline $\mathrm{R}(\mathrm{t})[1]$ & $\begin{array}{l}\mathrm{R}(\mathrm{t}) \text { CCFs } \\
{[2]}\end{array}$ & $\begin{array}{l}\text { PFD } \\
{[3]}\end{array}$ & $\begin{array}{l}\text { PFD_CCFs } \\
{[4]}\end{array}$ & $\begin{array}{l}\text { Ratio } \\
{[1] /} \\
{[2]}\end{array}$ & $\begin{array}{l}\text { Ratio } \\
{[4] /} \\
{[3]}\end{array}$ \\
\hline 0.4272 & 0.1676 & 0.4838 & 0.8303 & 2.548 & 1.716 \\
\hline
\end{tabular}

-The ratio: $\frac{[1]}{[2]}=\mathbf{2 . 5 4 8}$ is the factor of reduction of reliability.

-The ratio: $\frac{[4]}{[3]}=\mathbf{1 . 7 1 6}$ is the value of increasing PFD $_{\text {avg. }}$

Table 9 Evaluation ofthe HIPPS unconditional intensity: $w(t)$.

\begin{tabular}{|c|c|c|}
\hline $\begin{array}{c}\mathrm{w}(\mathrm{t}) \text { without } \\
\text { CCFs [1] }\end{array}$ & $\begin{array}{c}\mathrm{w}(\mathrm{t}) \text { with CCFs } \\
{[2]}\end{array}$ & Ratio [2]/ [1] \\
\hline $2.0423 \mathrm{E}-7$ & $6.3688 \mathrm{E}-6$ & 24.069 \\
\hline
\end{tabular}

increasing the accident frequency. Therefore, the CCF increases the frequency of the feared event.

\subsection{Discussion}

The simulation results show that the CCFs are a part of dependent failures which occurs in redundant HIPPS components (transmitters and valves), and it is a serious threat of the global HIPPS reliability. This kind of failure contributes too many major negative impacts: reduction of the global HIPPS reliability, increasing the PFD ${ }_{a v g}$, and the frequency of accident. Therefore, it is very necessary to implement measures for reducing the CCFs influence by the decrease of the Beta-factor using certain mechanisms.

\section{CONCLUSION}

In this paper the Fault Tree Analysis (FTA) method have been used for the study of the HIPPS (high integrity pressure protection system, in two cases with consideration of Common Cause Failures (CCFs) and without CCFs. In the first studies, we have calculated the different parameters of dependability:

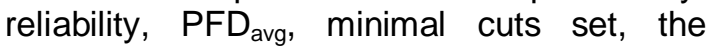
frequency of the feared accident, and the different importance factors that are an efficient tool to support decision making in reliability studies, and risk analysis, maintenance optimization for the HIPPS. These measures can be used for systems with binary components, and independent failures. For the second studies, we have also calculated the same various parameters (reliability, $P F D_{\text {avg }}$, frequency of accident) in the objective to make comparison between the two studies.

The simulation results show that the CCFs have a negative impacts on the global HIPPS

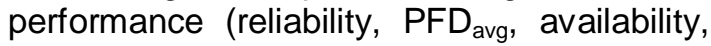
frequency of the accidents, production capacity......etc.), the HIPPS is a safety instrumented system provides several advantages such as flexibility and diagnostics abilities but at the same time there are many challenges when using this system. These challenges are due to dependencies as result of common design methods, the same environment (high or low temperature, large vibrations, high humidity, heat radiation, high or low pressures, electrical failure, corrosion, erosion ....etc.), operation, and maintenance procedures(failure to follow repair or test procedure, systematic calibration failures, inadequate testing /inspection ...etc.), which are the basic reason of CCFs.

The majors' defenses against CCFs are:

1) Physical separation of the main components.

2) Diversity of the redundancies.

3) Assessment/analysis and feedback of data.

4) Competence training.

5) Environmental control.

For the further work, we propose the CCFs implementation by the dynamic fault tree analysis (FTA) method, using the ALTARICA software.

\section{REFERENCES}

[1] International Electrotechnical Commission (IEC). Standard IEC61508: 2010, $2^{\text {nd }}$ edition functional safety of the Electrical /Electronic/Programmable Electronic SafetyRelated Systems.Geneva.

[2] P.Hokstard and M.Rausand "Common cause failure modeling: Status and trends". In Misra $\mathrm{KB}$, editor. Handbook for perfomability enginnering. London : Springer, pp.621640,2008 .

[3] NURG-75/014."'Reactor safety: an assessment of accident risk in the U.S commercial nuclear power plants".WASH1400.Wachington,DC,U.S.Nuclear Regulatory Commission, 1975.

[4] G.Edwards and A.Waston."A study of common-mode failures".1979,SRD R146,Wigahaw Lane, Culcheth Warrington (Wa34NE), United Kingdom Atomic Energy Authority.

[5] M.Rausand and A.Hoyland."System reliabilty, theory, models, statistical methods, and applications(second eddition), New York,2004.

[6] T.Lilleheier, "Analysis of common cause failure for complex safety instrumented systems". Master 's thesis, the Norwegian university of science and technology, Trondheim,2008. 
[7] L.Y.Wang."Common cause failure modeling in risk and reliabilty assessments: An evaluation of appraoches" Prpjet thesis RAM S group.NTNU,2011.

[8] M.Rausand, "Risk assessment theory, methods, and applications". Wiley, Hobken, NJ,2011.

[9] X.Z.W.Kuo, 'Importance measures in reliabilty risk and optimization: Principles and applications", W,S,Ltd, Ed.Wiley\&Sons Ltd,2012.

[10] X.Z.W.Kuo,"'Some recent advances on importance measures in reliabilty", IEEE Trans Reliability, vol.61,pp.344-360,2012.

[11] X.Z.W.Kuo,"'Relations and generalizations of importance measures in reliabilty", IEEE Trans Reliability, vol.61(3),pp.659-674,2012.

[12] API 170, "Recommended practice for subsea high Integrity Pressure Protection System(HIPPS)",American Petroleum Institute,2009.

[13] L.B.H.S.Roald Sirevaag, writer,experience with HTHP Subsea HIPPS on Kristin.[Performance].Statoil,2006.

[14] B.H.S.Jcob,G.Hoseth, "Optimizing Pressure in Subsea Piplines with "HIPPS".ABB review,no.2.pp.28-35,2000.

[15] P.T.R.S.G.Gail, "Reliability of Subsea control systems: HIPPS a case study", SCADA,pp.55-70,2002.
[16] J.P.Signoret,"'Methodology SIL evaluations related to HIPPS"-Total Draft Memo,2005.

[17] R.S.H.S.Lars Bak, "HIPPSprotects Subsea production in HT/HP conditions", offshore magazine,no.1,2007.

[18] E.W.Jacob and G.Hoseth, "Implementation options for the Subsea High Integrity Pipline Protection System (HIPPS) solution", in offshore Technology conference, Houston,1997.

[19] H.Ji,M.A.Lundteigen, .Rausand, 'Reliabilty performance of safety instrumented systems: A common approach for both low and high -,
demand mode ofoperation Reliabiltyenginneringandsafety vol96,pp.365373,2011.

[20] Y.Liu and ..Rausand ,"'Reliabilty assessment of safety instrumented systems subject to different demand modes". Journal of Loss Prevention in the Process Industries, vol24,pp.49-56,2006.

[21] M.Schonbeck, M.Rausand,Jan.Rouvroye, "Human and organisational factors in the operational phase of safety instrumented systems: A new approach". Safety science vol 48,pp.310-318,2010.

[22] OREDA Reliabilty Data Participants, Available from: Det Norske. Véritas, NO1322 Hovik, Norway,4rd edition, 2002. 